\title{
In Vitro Dusting Performance of a New Solid State Thulium Laser Compared to Holmium Laser Lithotripsy
}

\author{
Ralf Petzold, MD, MSc, Arkadiusz Miernik, MD, and Rodrigo Suarez-lbarrola, MD, BSc
}

\begin{abstract}
Purpose: To examine the dusting performance of a novel solid state Thulium laser device compared to a standard holmium:yttrium-aluminum-garnet (Ho:YAG) device.

Methods: This study compares a Dornier Medilas H Solvo 35 with an evaluation model of a pulsed solid state thulium:yttrium-aluminum-garnet (Tm:YAG) laser (Dornier MedTech Laser GmbH, Wessling, Germany). The in vitro model consisted of a mold with irrigated water at $37^{\circ} \mathrm{C}$. For $2-9$ minutes, laser fibers were guided by an xy-plotter in spirals over BegoStones. Stone mass was measured before and after laser application. Comparisons to Ho:YAG and further Tm:YAG investigations were performed.

Results: Identical settings with similar pulse durations yielded a significant 14\% advantage for Ho:YAG in slow fiber speeds and a tendency toward $15 \%$ higher efficiency of Tm:YAG in fast fiber speeds. Increased pulse duration in Tm:YAG led significantly to 32\%-54\% higher ablation rates in comparison to Tm:YAG in both setups. Ablated mass loss range is $102-1107 \mathrm{mg}$ for slow fiber speeds and 22-528 $\mathrm{mg}$ for fast speeds. Mass loss is proportional to pulse energy, frequency, and pulse duration, whereas pulse energy defines the penetration depth into the model stones. Frequency characterizes the ablation homogeneity and possible working speeds.

Conclusion: Tm:YAG is significantly more efficient when longer pulse durations are used. Identical settings revealed a strong connection to fiber movement speeds. In addition, the Tm:YAG device enables a broader range of settings with the possibility of minimal pulse energy of $100 \mathrm{~mJ}$ for low retropulsion and fine dusting with possible frequencies $\leq 200 \mathrm{~Hz}$.
\end{abstract}

Keywords: dusting, laser lithotripsy, Tm:YAG, Ho:YAG

\section{Introduction}

$\mathbf{H}$ OLMIUM:YTTRIUM-ALUMINUM-GARNET (Ho:YAG) laser lithotripsy originated in the early $1990 \mathrm{~s}^{1}$ and has evolved to become the gold standard in endoscopic stone treatment. ${ }^{2}$ Laser power settings have increased as much as $140 \mathrm{~W},{ }^{3}$ which have also improved efficiency with even more sophisticated pulse modulation technologies. ${ }^{4}$ In the shadow of Ho:YAG laser lithotripsy, thulium fiber lasers (TFLs) have been investigated for more than 15 years and shown comparable results for stone ablation and low retropulsion. ${ }^{5-7}$

The wavelength of thulium devices is slightly shorter than the Ho:YAG laser's, which comes with an increased absorption coefficient in water. ${ }^{8}$ A higher absorption coefficient speaks for a shorter penetration/absorption distance in water, less absorption time and higher energy density at the laser fiber tip. To objectively investigate how these physical consider- ations vary between laser types and influence dusting performance, laser devices should, therefore, be compared with similar energy and pulse duration settings.

In this study, an evaluation model of a pulsed thulium diode-pumped solid state/thulium:yttrium-aluminum-garnet (Tm:YAG) laser and a H35 Ho:YAG laser device (both by Dornier MedTech Europe $\mathrm{GmbH}$, Wessling, Germany) were compared. The Tm:YAG device uses a novel technology different to TFL and thulium constant wave technology. Furthermore, the Tm:YAG device offers $120 \mathrm{~W}$ of power with frequencies of as much as $200 \mathrm{~Hz}$ and possible single pulse energies as low as $100 \mathrm{~mJ}$. Regarding physical parameters, the Solvo H35's wavelength is $2080 \mathrm{~nm}$, and that of the Tm:YAG laser is $2013 \mathrm{~nm}$, which means that the Tm:YAG device's absorption coefficient in water is $84 \%$ higher $\left(3198 \mathrm{~m}^{-1}\right.$ vs $5888 \mathrm{~m}^{-1}$, at $37^{\circ} \mathrm{C}$ and 1013 mbar).

Department of Urology, Faculty of Medicine, University of Freiburg-Medical Centre, Freiburg, Germany. 


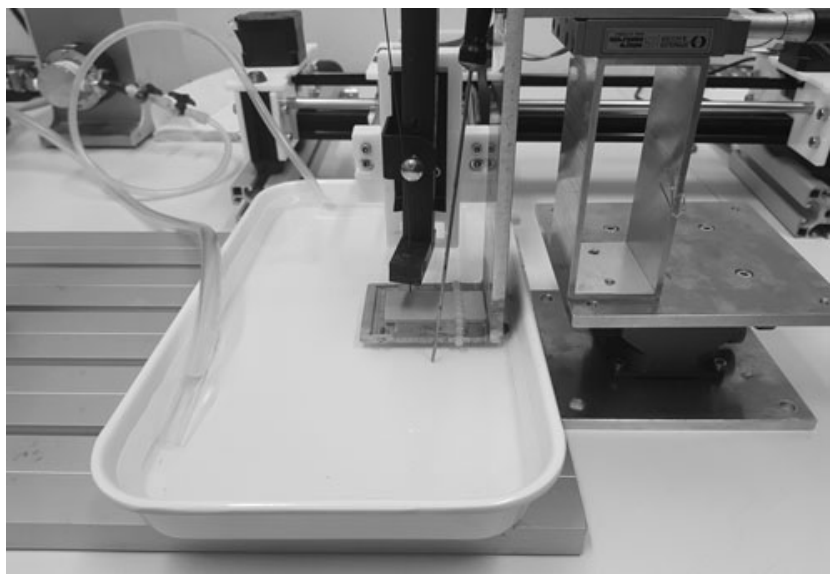

FIG. 1. Main part of the experimental setup.

The primary aim of our study was to compare the dusting efficiency of the examined devices with comparable settings. The secondary aim was to investigate the power and frequency limits of the new Tm:YAG device.

\section{Materials and Methods}

We partially replicated the setup described by Winship et al., where the laser fiber was moved over a stone model in a reproducible manner by stepper motors, and measuring mass loss afterward. ${ }^{9}$ We also used BegoStones as a stone model in a mixture of $100 \mathrm{~g}$ BegoStone powder with $22 \mathrm{~mL}$ water. The BegoStone mass was vibrated in a flat mold, rendering it largely free of bubbles, using a SL-JT51B vibration device (Guangzhou Sunlight Electronic Technology Co., Ltd., Guangzhou, China). The resulting stone plates were cut into $2.5 \times 6 \mathrm{~cm}$ pieces about $4 \mathrm{~mm}$ thick. The BegoStones were soaked in water for at least 24 hours before the experiments.

Our experimental setup consisted of a heated water bath, which was kept constant at $37^{\circ} \mathrm{C}$ with an Eheim heating rod thermocontrol 3604 (Eheim, Deizisau, Germany). The water bath's temperature was verified using a Pico Type K temperature sensor, which was evaluated with a USB TC 08 evaluation unit (PICO Technology, Cambridgeshire, UK). The values were read with MATLAB ${ }^{\circledR}$ (The Mathworks, Inc., Natick, MA) and displayed in real time.

A precision pump (Cole Parmer, Chicago, IL) was used to set a clinically realistic flow rate of $50 \mathrm{~mL} /$ minutes from the water bath to the test mold, which was directed close to the laser fiber tip. A $2.5 \mathrm{~cm}$ water level was kept constant by a hose pump (Samed GmbH, Dresden, Germany). The main part of our experimental setup is shown in Figure 1, including plotter, $400 \mu \mathrm{m}$ laser fiber and fiber mounting, and a constructed xyz leveling unit holding the BegoStone plate.

The laser power output was checked with an Ophir StarBright power meter (Ophir, Jerusalem, Israel) three times at the beginning of each test series and again before each individual test. The laser fiber was fixed to a 2D plotter (Eleks, Lviv, Ukraine). For the first test run, a spiral with 100 turns and $20 \mathrm{~mm}$ diameter was chosen as a sample, which was run at a constant speed of $500 \mathrm{~mm} /$ minutes starting from the inside. Five 9-minute runs were carried out per setting. The spiral's windings overlap by about $50 \%$, so that the material is dug away and not broken off at the edges.

In the second series of tests, a $20 \mathrm{~mm}$ diameter spiral created by 20 turns was run at a speed of $1500 \mathrm{~mm} /$ minutes. Hereby, the circular windings overlapped only minimally and because of increased fiber speed, each laser pulse created a separate crater. A quarter of the two intended spirals is shown in Figure 2I and II, in which the resulting ablation area of each experimental setup is shown.

To objectively assess the dusting performance, we measured mass loss as the difference in weight before and after the experiment with a precision balance EMB 200-2 (Kern $\&$ Sohn GmbH, Balingen, Germany). For this purpose, each stone was dabbed with cellulose paper before weighing so that there was no excess water on its surface. The measuring procedure was carried out within a few seconds since stones can quickly lose several milligrams of weight through evaporation. In addition, the particle size of the obtained dust was evaluated with sieves of decreasing aperture sizes such as 500,250 , and $125 \mu \mathrm{m}$. The water containing dust particles was poured through each sieve after the experiments to objectively determine the particles' sizes.

The investigation consisted of two parts. We made direct comparisons to Ho:YAG as well as additional experiments to evaluate the limits of the new device. The settings represent typical clinical dusting preferences and are listed in Table 1. Because of devices' limitations concerning possible pulse durations, we provide two direct comparisons with similar pulse durations.

Each test was repeated five times, the mean and standard deviation were determined and a student's $t$ test was carried out to examine different runs, using Microsoft Excel (Microsoft Corporation, Redmond). $p$-Values $<0.05$ were defined as statistically significant. The study involved no patients. Therefore, the need for Institutional Review Board approval was waived.
FIG. 2. Experimental results of $20 \mathrm{~mm}$ dusting spirals with the Thulium device, (I) slow, 100 converging circles and (II) fast, 20 nonconverging circles resulting in single craters.
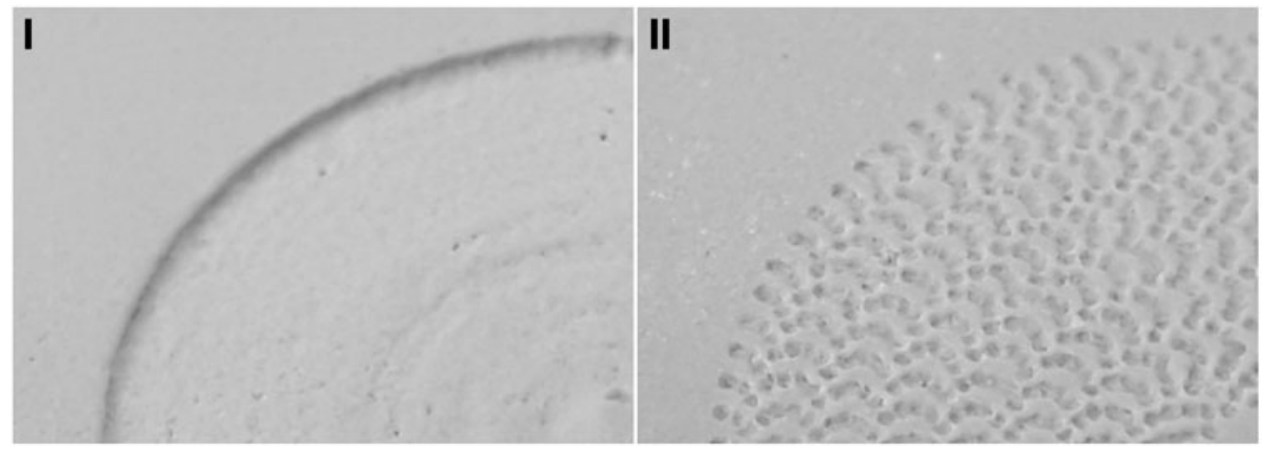


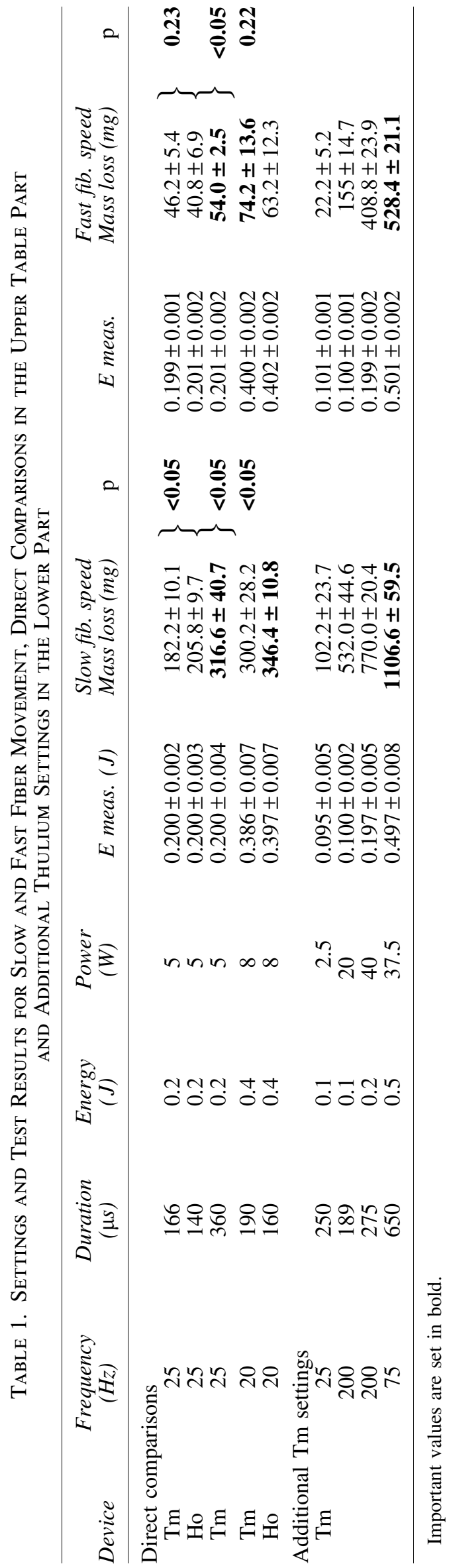

\section{Results}

Direct comparisons between Tm:YAG and Ho:YAG revealed significantly higher ablation rates for Ho:YAG laser at slow speed $(p<0.05)$. The average ablation at $400 \mathrm{~mJ}$ and $20 \mathrm{~Hz}$ was $300 \mathrm{mg}$ for thulium and $346 \mathrm{mg}$ for Ho:YAG, equivalent to ablation rates of 556 and $641 \mu \mathrm{g} / \mathrm{seconds}$, respectively. At $200 \mathrm{~mJ}, 25 \mathrm{~Hz}$, and similar pulse durations the corresponding values were $182 \mathrm{mg}$ for Tm:YAG and $206 \mathrm{mg}$ for Ho:YAG, equaling 337 and $381 \mu \mathrm{g} / \mathrm{seconds}$, respectively. With a pulse duration of $366 \mu \mathrm{s}$ at $200 \mathrm{~mJ}$ and $25 \mathrm{~Hz}$, the Tm:YAG device showed a $74 \%$ mass loss increase in comparison to a pulse duration of $166 \mu$ s, significantly outperforming Ho:YAG by $54 \%$ and improving the ablation rate to $586 \mu \mathrm{g} / \mathrm{seconds}$. Similar findings were obtained for the pulse duration's influence in Tm:YAG for the fast fiber speed. The results are presented in Table 1.

The Tm:YAG laser's additional settings resulted in ablation values directly dependent on frequency and single pulse energy. However, a nonlinear correlation was obtained, for example, $102 \mathrm{mg}$ at $100 \mathrm{~mJ} / 25 \mathrm{~Hz}$ and $532 \mathrm{mg}$ at $100 \mathrm{~mJ} / 200 \mathrm{~Hz}$, which corresponds to $a+522 \%$ increase in the ablation rate in a power increase of $+800 \%$. We measured a maximum ablation of $1107 \mathrm{mg}$ for the $500 \mathrm{~mJ}$ and $75 \mathrm{~Hz}$ setting, corresponding to $2050 \mu \mathrm{g} / \mathrm{seconds}$.

The Ho:YAG and Tm:YAG comparison results with the faster fiber movement revealed a tendency toward more efficient ablation for the Tm:YAG laser; however, this finding was not significant in all five experiments that we performed. The faster fiber speed experiments delivered mass losses, which corresponded to the $500 \mathrm{~mm} /$ minute experiments, whereby a strong dependence between power and ablation was similarly evident.

Concerning particle size, we were unable to determine their size using the sieves since the generated dust was finer than $125 \mu \mathrm{m}$.

\section{Discussion}

We found a significant and 13\%-15\% more effective ablation rate for the holmium device when compared to the Tm:YAG at a slow speed of $500 \mathrm{~mm} /$ minute $(p<0.05)$ using almost identical energy/frequency settings and similar pulse durations. Changing the Tm:YAG pulse duration from 166 to $360 \mu$ s yielded a $74 \%$ increase in otherwise identical settings, giving Tm:YAG a significant 54\% advantage over Ho:YAG.

At a fast speed of $1500 \mathrm{~mm} /$ minute, the Tm:YAG device showed a tendency toward a 12\%-17\% higher effectiveness, although this was not statistically significant. Likewise, a longer Tm:YAG pulse duration offered higher ablation rates and a $32 \%$ advantage over Ho:YAG.

According to the results reported by Blackmon et al., ${ }^{5}$ we expected a higher impact of differing wavelengths in the two technologies, which could not be confirmed. It is plausible that this may be attributable to the smaller differences in wavelengths of Tm:YAG and Ho:YAG in comparison to TFL.

In addition to our direct comparisons, the experiments we ran to assess the limits of the new Tm:YAG laser yielded promising results. Particularly, $200 \mathrm{~Hz}$ frequencies coupled to low $100 \mathrm{~mJ}$ pulse energies presumably enable efficient fine dusting at low retropulsion, which is currently not achievable with holmium lasers with a minimum of $200 \mathrm{~mJ}$ and maximum $80 \mathrm{~Hz}$ (e.g., Pulse $120 \mathrm{H}$ from Lumenis, San Jose). In the 
automated experiments with the $100 \mathrm{~mJ} / 200 \mathrm{~Hz}$ setting, we found that more than $500 \mathrm{mg}$ of BegoStone material was ablatable within the 120 seconds, which corresponds to $4167 \mu \mathrm{g} / \mathrm{seconds}$. The stone dust produced was fine enough to avoid collection in a $125 \mu \mathrm{m}$ sieve. This means that no additional extraction tools such as baskets would be needed and the stone dust can be cleared directly with the irrigation. We therefore suggest speaking of fine dusting if no residual fragment occurs and the particle size is below $125 \mu \mathrm{m}$.

By using the 2D plotter, we ensured that only dusting and no fragmentation occurs. Stone material was thus completely pulverized without leaving any particle larger than $125 \mu \mathrm{m}$. In everyday clinical practice with actual human kidney stones, partial fragmentation and residual fragments can hardly be avoided, since the natural crystalline structures of various types of kidney stones are heterogeneous and can form natural extensions that break off easily during laser lithotripsy. ${ }^{10}$

On the other hand, in contrast to BegoStones, the crystals and especially calcium oxalate stones provide natural fragmentation lines that cannot be influenced by fiber guidance. Dusting therefore inevitably results in larger fragments in everyday clinical practice, which may need individual recovering with baskets and other endourological tools. Since a kidney stone's fracture along natural fissures depends on the pulse energy, there is some evidence to suggest that low pulse energies could lead to delayed fracturing and thus smaller fragments being produced, which are ideal for spontaneous clearance through irrigation. To work efficiently with small pulse energies, high frequencies remain necessary, which are currently limited to $80 \mathrm{~Hz}$ in holmium technology. ${ }^{3}$ Solid state technology with $200 \mathrm{~Hz}$ therefore has enormous potential to enable efficient fine dusting.

Blackmon et al. investigated the ablation threshold, ablation rate, and retropulsion of a TFL. ${ }^{5}$ However, because of laser settings and wavelengths that differ from those of the solid state technology, our results are not directly comparable (TFL 1908/1940 nm vs solid state $2013 \mathrm{~nm}$ ). They found the TFL device to be superior to Ho:YAG in terms of dusting efficiency with minimal retropulsion because of its low single pulse energies. Specifically, holmium $165 \mathrm{~mJ} / 10 \mathrm{~Hz}$ and TFL $35 \mathrm{~mJ} / 100 \mathrm{~Hz}$ settings were compared and ablation rates of 100 and $140 \mu \mathrm{g} / \mathrm{seconds}$ were detected, respectively. In terms of power output, 1.65 $\mathrm{W}$ was compared to $3.5 \mathrm{~W}$; therefore, TFL can only achieve $a+40 \%$ higher ablation rate with $+112 \%$ higher power. In our study, we used powers starting at $5 \mathrm{~W}$ and we detected a maximum ablation rate of $586 \mu \mathrm{g} / \mathrm{seconds}$, which is fourfold above the $140 \mu \mathrm{g} / \mathrm{seconds}$ reported by Blackmon et al. at a power increase of only $43 \%$.

Several investigators have reported that an additional advantage of using low-power lithotripsy is the improved patient safety profile, provided sufficient irrigation is applied. ${ }^{11-14}$ High ablation rates at minimum power are thus desirable, which can be attained with both TFL and solid state technology since the shorter absorption distance requires less power for the same energy density on the fiber's tip.

We demonstrated that the investigated devices produce similar results at similar settings and that the Tm:YAG device's potential for efficient fine dusting is high. Concerning usability, the Tm:YAG device was similar to the Ho:YAG device and showed no laser failure. Considering the clinical application, we assume that the new technology will allow a more convenient workflow and reduced operating time attributable to the increased range of power and frequency settings to accommodate to the different needs during dusting and fragmenting. Reduced pulse energy and long pulse duration lead to less retropulsion, which may lead to a more controlled and precise dusting, leaving fewer fragments to be retrieved individually.

Nevertheless, there are limitations in this study that affect the applicability of our findings. For example, the dusting performance under laboratory conditions was investigated in an in vitro experiment in which ambient conditions are controllable. It was not possible to use identical pulse durations because of technical limitations of examined laser devices. We observed a quick fiber degradation starting with the first pulses in both devices. Even though fibers were cut or replaced after each experimental run, this is a bias when comparing the two fiber speeds with different durations of the experiments. The BegoStone models we used have a fundamentally different composition and structure than real human kidney stones.

We can conclude that particle size was below $125 \mu \mathrm{m}$, but were unable to detect an exact particle size; therefore, an electron-microscopic evaluation on this topic will be conducted in further research. We also used an xy-plotter, which, with the BegoStone plate clamped in place, allows reproducible results to compare different devices. However, in everyday clinical practice, many more environmental variables play a role in affecting the efficiency of lithotripsy. Therefore, further research on this novel laser device should be conducted to investigate its clinical performance.

\section{Conclusion}

The new Tm:YAG solid state laser device's dusting performance proved to be similar to that of the Ho:YAG laser device at similar settings. The additional advantages of longer pulse duration, high frequencies, and low single pulse energies delivered promising results, namely highly efficient fine dusting in conjunction with low retropulsion. The Tm:YAG device significantly outperformed the Ho:YAG device by offering longer pulse durations in otherwise similar settings. A higher fiber movement speed showed a tendency toward increased ablation effectiveness in Tm:YAG.

\section{Author Disclosure Statement}

No competing financial interests exist. R.P and A.M were consultants to Dornier MedTech Laser GmbH, Wessling, Germany, purely for the sake of this investigation.

\section{Funding Information}

No funding was received for this study.

\section{References}

1. Johnson DE, Cromeens DM, Price RE. Use of the holmium:YAG laser in urology. Lasers Surg Med 1992;12:353363.

2. Oberlin DT, Flum AS, Bachrach L, Matulewicz RS, Flury SC. Contemporary surgical trends in the management of upper tract calculi. J Urol 2015;193:880-884.

3. Fried NM. Recent advances in infrared laser lithotripsy. Biomed Opt Express 2018;9:4552-4568. 
4. Elhilali MM, Badaan S, Ibrahim A, Andonian S. Use of the moses technology to improve holmium laser lithotripsy outcomes: A preclinical study. J Endourol 2017;31:598-604.

5. Blackmon RL, Fried NM, Irby PB. Comparison of holmium: YAG and thulium fiber laser lithotripsy: Ablation thresholds, ablation rates, and retropulsion effects. J Biomed Opt 2011; 16:071403.

6. Blackmon RL, Irby PB, Fried NM. Holmium: YAG (wavelength $=2120 \mathrm{~nm}$ ) vs. Thulium fiber laser (wavelength $=1908 \mathrm{~nm}$ ) ablation of kidney stones: Thresholds, rates, and retropulsion. In: Photonic Therapeutics and Diagnostics VII: International Society for Optics and Photonics, 2011, p. 788318.

7. Blackmon RL, Irby PB, Fried NM. Improved thulium fiber laser vaporization of urinary stones using micro-pulse packets. In: Photonic Therapeutics and Diagnostics VIII: International Society for Optics and Photonics, 2012, p. 82071C.

8. Teichmann HO, Herrmann TR, Bach T. Technical aspects of lasers in urology. World J Urol 2007;25:221-225.

9. Winship B, Wollin D, Carlos E, Li J, Peters C, Simmons WN, et al. Dusting efficiency of the moses holmium laser: An automated in vitro assessment. J Endourol 2018;32: 1131-1135.

10. Hardy LA, Irby PB, Fried NM. Scanning electron microscopy of real and artificial kidney stones before and after Thulium fiber laser ablation in air and water. In: Therapeutics and Diagnostics in Urology 2018: International Society for Optics and Photonics, 2018, p. 104680G.

11. Hein S, Petzold R, Schoenthaler M, Wetterauer U, Miernik A. Thermal effects of Ho: YAG laser lithotripsy: Real-time evaluation in an in vitro model. World J Urol 2018;36: 1469-1475.
12. Molina WR, Silva IN, Donalisio da Silva R, Gustafson D, Sehrt D, Kim FJ. Influence of saline on temperature profile of laser lithotripsy activation. J Endourol 2015;29: 235-239.

13. Buttice S, Sener TE, Proietti S, Dragos L, Tefik T, Doizi S, et al. Temperature changes inside the kidney: What happens during holmium:yttrium-aluminium-garnet laser usage? J Endourol 2016;30:574-579.

14. Hein S, Petzold R, Suarez-Ibarrola R, Müller P-F, Schoenthaler M, Miernik A. Thermal effects of Ho: YAG laser lithotripsy during retrograde intrarenal surgery and percutaneous nephrolithotomy in an ex vivo porcine kidney model. World J Urol 2020;38:753-760.

$$
\begin{array}{r}
\text { Address correspondence to: } \\
\text { Ralf Petzold, MD, MSc } \\
\text { Department of Urology } \\
\text { Faculty of Medicine } \\
\text { University of Freiburg-Medical Centre } \\
\text { Hugstetter Street } 55 \\
\text { Freiburg D-79106 } \\
\text { Germany }
\end{array}
$$

E-mail: ralf.petzold@uniklinik-freiburg.de
$\begin{aligned} & \text { Abbreviations Used } \\ & \text { Ho: } Y A G=\text { holmium:yttrium-aluminum-garnet }\end{aligned}$
$\mathrm{TFL}=$ thulium fiber laser
Tm:YAG = thulium:yttrium-aluminum-garnet 